\title{
Challenging pretreatment aspects of lung cancer in Georgia
}

\author{
Ivane Kiladze ${ }^{1 *}$, Elene Mariamidze', Branislav Jeremic ${ }^{2}$
}

'Oncology and Hematology Department, Research Institute of Clinical Medicine after academician F.Todua, Tbilisi, Georgia ${ }^{2}$ Radiation Oncology Department, Research Institute of Clinical Medicine after academician F.Todua, Tbilisi, Georgia

\section{Article Info}

\section{Article Notes}

Received: September 21, 2020

Accepted: October 26, 2020

\section{*Correspondence:}

Dr. Ivane Kiladze, Oncology and Hematology Department, Research Institute of Clinical Medicine after academician, F.Todua, Tbilisi, Georgia; Email: ivane6@hotmail.com

(c) 2020 Kiladze I. This article is distributed under the terms of the Creative Commons Attribution 4.0 International License.

\section{Keywords}

Lung cancer

Screening

Prevention

Early diagnostics

Staging

Georgia

\section{Abstract}

Lung cancer (LC) continues to be a significant worldwide public health issue. There are several publications addressing specifics of $L C$ worldwide, but none concerning Georgia- a country with high number of smoking population and LC cases.

Based on the above facts we conducted the first study in the country that aims both evaluating current pretreatment LC challenges, including barriers for early diagnostics and indicating the future strategies for improved LC care.

We first analyzed LC statistics and the smoking patterns in the country. Further, we identified other challenging issues in pretreatment diagnostics and staging aspects and finally, provided a survey among LC specialists all over the country to evaluate the situation regarding access and use of radiology investigations and other staging procedures. The survey questionnaire was distributed among LC specialists in main cancer hospitals $(n=13)$ across the country.

We identify multiple health challenges. Still there are a high number of smokers in the country which clearly indicates that additional measures focusing on smoking cessation are urgently needed. This is further materialized in the fact that the majority of patients with LC are diagnosed with either locally advanced or metastatic disease. Activation of preventive programs and implementation of LC screening for early detection should hopefully lead to further reduction of national LC mortality rates.

We underline the urgent need for implementation of country-adapted diagnostic and therapeutic guidelines and protocols as well as enforcing multidisciplinary team meetings. The great need to introduce screening programs in high risk groups, improve access to modern treatment modalities and standardize national diagnostic and treatment protocols are of paramount importance for better LC care.

\section{Introduction}

Cancer is a leading cause of death worldwide, accounting for an estimated 9.6 million deaths in 2018. Globally, about 1 in 6 deaths is due to cancer. Approximately $70 \%$ of cancer deaths occur in lowand middle-income countries ${ }^{1}$. Lung cancer (LC) has been the most common malignancy and leading cause of cancer death worldwide ${ }^{2}$. The global incidence of lung cancer is increasing: in 2018, there were 2 million new cases, an increase from 1.8 million new cases in 2012. LC is more common in men, among whom the highest incidence rates are in Central and Eastern Europe, along with Eastern $\mathrm{Asia}^{3}$.

Georgia is a country located at the crossroads of Western Asia and Eastern Europe with just over 3.7 million citizens ${ }^{4}$. The incidence of LC alone accounts for $13 \%$ in both sexes of Georgian population, with male gender predominance which accounts for $20 \%$ of all 
cancers in men ${ }^{5}$. In Georgia, only about $10 \%$ of the new cases of LC are diagnosed at early stages (stage I-II) and $60 \%$ fall into stage $\mathrm{IV}^{6}$, significantly influencing diagnostic and treatment approaches as well overall treatment results. These alarming data call for an urgent shift of interests towards cancer prevention, screening, and early diagnostics, as it was shown that there is A big difference of survival between stage I ( $>90 \%)^{7}$ and stage IV disease, being of paramount important for early diagnostics of LC.

Importantly, too, The National Lung Screening Trial (NLST) was the first study well powered enough to show decrease in LC-specific mortality which was confirmed on their recent extended follow up ${ }^{8}$, as well the NELSON results were just published confirming the benefit to LC screening 9 .

The Georgian health care system is divided into a public and a private sector, with coverage of nearly $100 \%$ of the population. In the health plans, the patients have a great deal of freedom in choosing their physicians in most specialties and hospitals in most areas of the country. The Basic Package of the Universal Health Care Program launched in 2013 includes the treatment of oncologic patients, specifically chemotherapy, hormone therapy and radiotherapy and investigations and medications related to these procedures. The program covers all types of laboratory and instrumental investigations related to planned treatment ${ }^{10}$. Importantly, since 2015 , population-based Cancer Registry was implemented in the country. The Registry plays a significant role in terms of gathering data on cancer incidence and prevalence in the country ${ }^{11}$.

Based on above facts we conducted the first study nationwide that aims to both evaluate current pretreatment LC challenges including barriers for early diagnostic as well as identify future strategies for improved LC care.

\section{Materials and Methods}

In our study, we first retrieved the National Center for Disease Control and public health (NCDC) statistics on LC. Then, we analyzed the smoking patters of our society and the role of governmental tobacco control plan on the reduction of smokers in the country. Further to it, we identified additional challenging issues in pretreatment diagnostics and staging aspects in LC. Finally, we surveyed LC specialists all over the country to evaluate the situation regarding access and the use of radiology investigations and other staging procedures. The survey questionnaire was distributed among surgical (thoracic surgeon), medical and radiation oncologists in main oncology hospitals $(n=13)$ across four largest cities of Georgia, where the vast majority of patients with LC are treated. The questionnaire contained both open-ended and single best choice answer questions (12 questions related to pretreatment management and staging procedures), with separate sections for non-small cell (NSCLC) and small cell (SCLC) cancers. Survey data was collected and descriptive statistics used to present the results. The study was internally reviewed during a regularly scheduled scientific development review at our center in the absence of an Institutional Review Board.

The survey went out to a total of 24 physicians representing medical oncology $(n=13)$, radiation $(n=7)$ and thoracic surgeon $(n=4)$ and all responded. All physicians were with more than 5 year experience, with 13 male and 11 female doctors. Except thoracic surgeons, all physicians are general oncologists.

\section{Results}

\section{Smoking epidemiology in Georgia}

Smoking is one of the modifiable risk factors attributing to $75-85 \%$ of LC cases. Most LCs could be averted by preventing smoking initiation among adolescents and increasing smoking cessation among adults. Variations in LC patterns largely reflect country-specific differences in the stage and degree of the tobacco epidemic ${ }^{12}$. Georgia has a high number of smokers, $31.1 \%$ of population [Figure 1]. Average age of starting smoking in males and in females is 17.8 years and 22.4years, respectively. The majority of smokers (98.6\%) smoke manufactured cigarettes, while25.3 \% of current smokers (male 25.5\%, female $24.2 \%$ ) tried to quit smoking during the past 1 year and $9.4 \%$ of those who have tried to quit smoking applied electronic cigarettes ${ }^{13}$. In 2017, the Parliament of Georgia adopted a new law on tobacco control. Public awareness campaign/activities about basic manageable risk factors (tobacco, alcohol, low physical activity, excess weight, obesity) is implemented within the State Health Promotion Program.

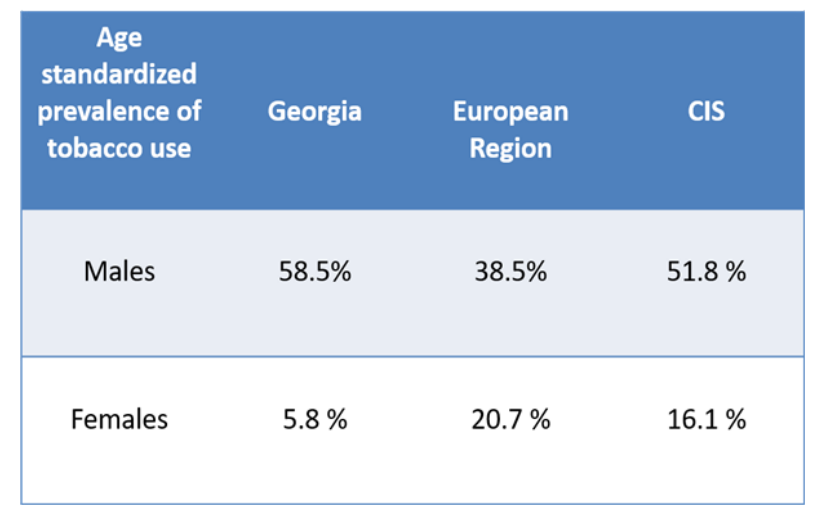

Source: Sturua L et al Smoking prevalence in Georgian adults: results of non-communicable disease risk factors STEPS 2016 survey. Tobacco Prevention \& Cessation. 2018; 4(Supplement): A166 Doi:10.18332/tpc/90457

Figure 1: Age standardized prevalence of tobacco use 


\section{Diagnosis and Staging}

There is currently no active LC public screening program for high-risk populations in the country. Vast majority of responders (23 out of 24) agree that starting LC screening program in Georgia will be beneficial, since diagnosing LC at an early stage will lead to more curative treatment options leading to a better survival.

All diagnostic and staging modalities exist in the country. While computed tomography (CT) scanners are available in all tertiary centers, magnetic resonance imaging (MRI) is available in many hospitals in the country. Nuclear medicine diagnostic procedures that have technetium $99 \mathrm{~m}$ (Tc-99m), and positron emission tomography (PET) are also available for cancer patients, with however, only two hospitals housing PET-CT machines, both services being located in capital, Tbilisi $^{10}$.

For initial staging procedures, all 13 institutions perform chest CT and the vast majority also perform abdominal CT (12 out of 13). The ways and tools for obtaining the biopsy specimen are still challenging country-wide as 9 out of 13 hospitals still consider exploratory thoracotomy as one of the options in the diagnosis and/or staging.

Wide variation was observed between hospitals in using PET-CT in non-small cell lung cancer (NSCLC) and small cell lung cancer (SCLC). In NSCLC, 4 hospitals (30\%) perform PET-CT only in patients who are surgical candidates while additional 5 (38\%) do so in locally advanced, unresectable disease [Figure 2]. In SCLC, 2 hospitals always suggest PETCT for routine staging while additional 5 (38\%) use it only in patients with limited disease, additional 2 use it in extensive disease and remaining 4 use it extremely rarely [Figure 3].

Seven hospitals out of 13 consider initial brain MRI in all patients with SCLC to rule out brain metastases. For NSCLC, indication for brain MRI in 6 institutions' (46\%) is locally advanced disease, while the same number (46\%) consider it only in symptomatic patients.

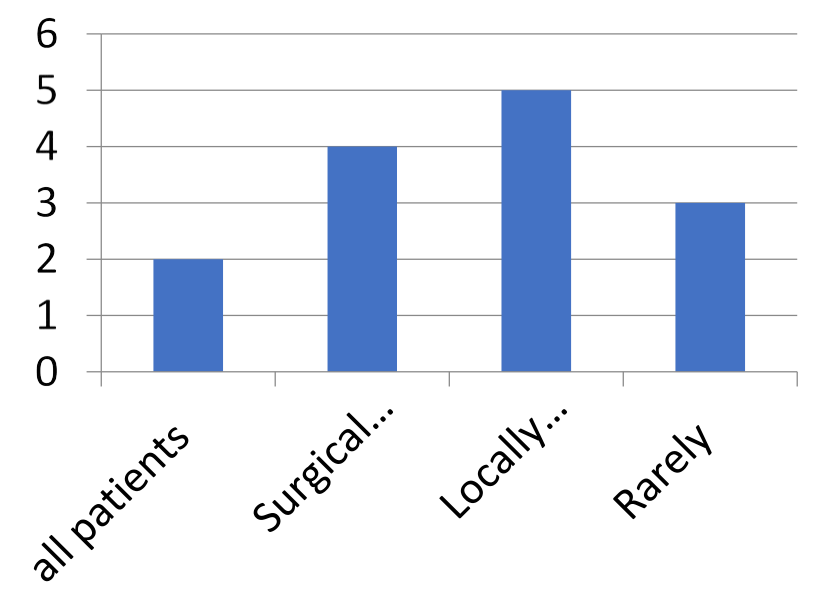

Figure 2: PET-CT in NSCLC

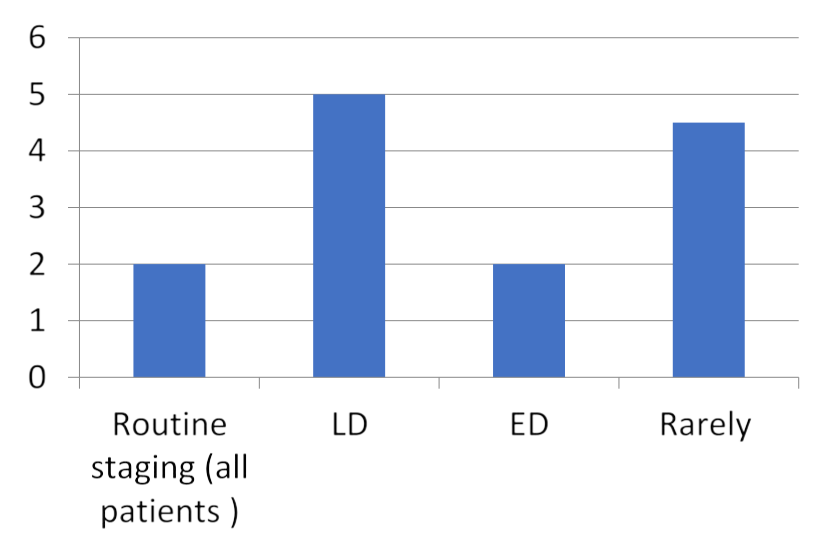

Figure 3: PET-CT in SCLC

Histologic/cytologic examination is done by bronchoscopy, including, if necessary, the endobronchial ultrasound (EBUS) procedure or CT-guided biopsy. EBUS is available both for biopsy/staging only in one hospital. There is no EUS /FNA (endoesophageal ultrasoundguided fine needle aspiration) in the country. Only in few hospitals VATS is available for staging and biopsy procedures. Percutaneous transthoracic biopsy, performed by interventional radiology is not available in all hospitals.

Once malignancy has been confirmed on pathological examination, the diagnosis of SCLC or NSCLC based on morphologic features is determined and a full panel of diagnostic biomarkers is available in questionable cases. The rise of targeted therapy and immunotherapy helped testing of tumors for present genetic mutations to become increasingly important. All biomarker testing is available in the country, but not reimbursed, with only exception of testing for epidermal growth factor receptor (EGFR) oncogenic mutation ${ }^{14}$. In majority cases, biomarker testing is performed after recommendation from the responsible oncologist, but it is not routinely used due to limited access modern therapeutics. In everyday clinical practice all medical oncologists $(n=13)$ consider molecular testing for EGFR in adenocarcinoma histology, while 9 out of 13 consider PD-L1 testing for NSCLC despite histology type. Eight and seven out of 13 oncologists consider testing for ALK and ROS1 respectively. Waiting time from initial diagnostics to starting treatment in 11 out of 13 hospitals is 2-4 weeks. In all but one hospital patients are encouraged to stop smoking during the treatment.

\section{Discussion}

It is well known that within the next 10 years, $70 \%$ of patients with newly diagnosed cancer will be living in countries that collectively have only $5 \%$ of the global resources for cancer control. Importantly, approximately $60 \%$ of the world's cancer patients presently do not have access to all novel drugs for systemic therapy and even more for radiotherapy (RT) ${ }^{15}$. Georgia, a country with its 
limited resources, faces multiple challenges in pretreatment assessment as well as access to treatment.

Our study aims both evaluating current lung cancer diagnostics and staging challenges and indicating the future strategies for improved cancer care in the country. Information was collected from institutions which are adequately equipped for diagnostic purposes, as well as providing "modern" treatment, with thoracic surgery department $(\mathrm{N}=4)$, RT $(\mathrm{N}=6)$ while all of them having medical oncology $(n=13)$ services. Surgeons whose opinion and practices were included in this survey were those with experience in the diagnosis and treatment of LC and who, importantly, are actively involved in multidisciplinary team (MDT) meetings in LC. With this approach, we tried to secure our data reflecting the national realities in the LC diagnosis and pretreatment staging.

Lacking screening programs present as the first obstacle, likely leading to significant under diagnosis and missing early stage patients. In our survey, the vast majority of physicians working in the field of LC agree that screening program will be very useful and important for the country, for early detection of cancer leading to a better survival.

In the past decade LC screening trials such as the extended follow up of the NLST and NELSON trial ${ }^{8,9}$ have confirmed a decrease in lung-cancer specific mortality in the USA and in Europe, while demonstrated a stageshift with increased identification of early and therefore treatable disease. Importance of LC screening was reported in 2019 by the American Lung Association's "State of Lung cancer", which showed dramatically increased survival rates. The 5-year survival rate is now $21.7 \%$, up from $17.2 \%$ a decade ago. This is a dramatic $26 \%$ improvement over the past 10 years, which the study group attributed to LC screening and advancements in LC research. As the data from the USA clearly showed, screening programs have substantial impact on the fate of LC patients with significant improvements in the overall results ${ }^{16}$.

Another large study of smokers and ex-smokers in the Netherlands and Belgium confirmed that widespread screening using low-dose CT scans can dramatically lower the LC death rate. After 10 years, the mortality rate for men and women who received regular screening was reduced by $24 \%$ and $33 \%$, respectively ${ }^{9}$.

Furthermore, use of nodule classification systems such as the Fleischer criteria or LUNG-rads may leads to less false positives. As LC screening is not currently established in our country, other barriers include whether our government would be willing to pay, establishing universally adopted screening criteria, as well as overcoming low adherence to protocols seen elsewhere.

Perhaps re-introducing the place and role of GPs in this scenario would be of additional help. Not to be forgotten, although state-controlled programs exist addressing the nation-wide problem of smoking, it is still far from being satisfactory and, hence, results are still lacking. In country with such high number of active smokers more strict control and more efforts must be implemented to reduce smoking, as tobacco use is the most important risk factor for cancer and is responsible for approximately $22 \%$ of cancer deaths ${ }^{17}$.

In the domain of diagnostics, except EUS/FNA, all investigations exist in the country, including PET-CT. However, there is no a cyclotron so the Fluorodeoxyglucosae (FDG) needs to be imported which limits access to it. Among responders, differences were observed on indication and time of brain MRI in both NSCLC and SCLC. Differences were also observed concerning indications for other radiology assessments. These results show need and imminent importance of development and implementation of nationally adapted and agreed upon guidelines and protocols. The country still faces significantly limited access to all molecular profiling platforms of the tumor and molecular testing generally is employed at the discretion of the treating oncologist.

In addition to the above, there are a number of challenges, similar to many, if not all, LMIC, such as low socioeconomic status, lack of public awareness, geographical (un)availability and fragmentation of health care services. It is well known that people with low socioeconomic status are more likely to engage in unhealthy behaviors, such as smoking. The association between low socioeconomic status and lung cancer emerges clearly in national statistics ${ }^{18}$.

\section{Conclusion}

In summary, the Georgian health service, despite noticeable improvements in recent years still faces multiple health challenges. Still, we have a high number of smokers in the country and the majority of patients with LC are diagnosed with either locally advanced or metastatic disease. Activation of preventive programs and implementation of LC screening for early detection should hopefully lead to further reduction of national LC mortality rates. Also, additional measures focusing on smoking cessation are extra important. We explore the urgent need for implementation of nationwide, country-adapted, diagnostic and therapeutic guidelines and protocols as well as enforcing MDT meetings. The great need to introduce screening programs in high risk groups, improve access to modern treatment modalities and standardize national diagnostic and treatment protocols are the steps of paramount importance steps for better LC care.

\section{Highlights}

Lung cancer (LC)- a significant worldwide public health issue. 
Especially low/middle income countries face challenges in diagnostics and treatment of LC.

The first study in the country that aims both evaluating current pretreatment LC challenges, including barriers for early diagnostics and indicating the future strategies for improved LC care.

\section{Findings}

- We identify multiple health challenges.

- High number of smokers in the country.

- The vast majority of patients with LC are diagnosed with either locally advanced or metastatic disease.

- Limitations in access to full spectrum staging procedures and molecular testing.

\section{Solutions}

- The great need to introduce screening programs in high risk groups.

- Additional measures focusing on smoking cessation are urgently needed.

- Improve access to modern treatment modalities and standardize national diagnostic and treatment protocols are of paramount importance for better LC care.

\section{- Enforcing MDT meetings.}

As a next step to improve lung cancer care it is planned to create "Lung Cancer Working Group" in the country. Our efforts will be directed towards collaborating with international lung cancer study groups.

\section{References}

1. WHO-Cancer -Fact sheets. https://www.who.int/news-room/factsheets/detail/cancer. Access 12 September 2018

2. Bray F, Ferlay J, Soerjomataram I, et al. Global cancer statistics 2018: GLOBOCAN estimates of incidence and mortality worldwide for 36 cancers in 185 countries. CA Cancer J Clin. 2018 Nov; 68(6): 394-424. doi: 10.3322/caac.21492. Epub 2018 Sep 12. Erratum in: CA Cancer J Clin. 2020 Jul; 70(4): 313. PMID: 30207593.

3. World Cancer Research Fund International, American Institute for Cancer Reasearch. Lung cancer statistics[Internet]. London: World Cancer Research Fund International; [cited 21 May 2019]. Available from: https://www.wcrf.org

4. Key Indicators. http://www.geostat.ge/index. php?action=0\&lang=eng . Accessed 16 Oct 2017.
5. Global Cancer Observatory. IARC. https://gco.iarc.fr/today/ data/factsheets/populations/268-georgia-fact-sheets.pdf. [Accessed May.2019]

6. NCDC. Population-based cancer registry. Georgia.2019. https:// www.ncdc.ge/Pages/User/News.aspx?ID=1f20368c-fbf4-40f1-8fd36fa2ad88a11e

7. International Early Lung Cancer Action Program Investigators, Henschke CI, Yankelevitz DF, Libby DM, et al. Survival of patients with stage I lung cancer detected on CT screening. N Engl J Med. 2006 Oct 26; 355(17): 1763-71. doi: 10.1056/NEJMoa060476. Erratum in: N Engl J Med. 2008 Apr 24; 358(17): 1875. Erratum in: N Engl J Med. 2008 Aug 21; 359(8): 877. Erratum in: N Engl J Med. 2008 Apr 24; 358(17): 1862. PMID: 17065637

8. National Lung Screening Trial Research Team. Lung Cancer Incidence and Mortality with Extended Follow-up in the National Lung Screening Trial. J Thorac Oncol. 2019 Oct; 14(10): 1732-1742. doi: 10.1016/j.jtho.2019.05.044. Epub 2019 Jun 28. PMID: 31260833; PMCID: PMC6764895.

9. de Koning HJ, van der Aalst CM, de Jong PA, et al. Reduced Lung-Cancer Mortality with Volume CT Screening in a Randomized Trial. N Engl J Med. 2020 Feb 6; 382(6): 503-513. doi: 10.1056/NEJMoa1911793. Epub 2020 Jan 29. PMID: 31995683.

10. Cancer Profile. Georgia. https://www.moh.gov.ge/uploads/ files/2018/Failebi/16.04.2018.pdf Accessed April.2018

11. National Center of Disease Control and Public Health http://www. ncdc.ge

12. WHO. Global action plan for the prevention and control of non communicable diseases 2013-2020. https://apps.who.int/iris/bitstream/ handle/10665/94384/9789241506236_eng.pdf?sequence=1

13. Sturua L, Maglakelidze N, Gamkrelidze A. Smoking prevalence in Georgian adults: results of non-communicable disease risk factors STEPS 2016 survey. Tobacco Prevention \& Cessation.2018; 4(Suppl): A166. Doi:10.18332/tpc/90457 https://www.who.int/ncds/ surveillance/steps/Georgia_2016_STEPS_FS.pdf

14. Kiladze I, Mariamidze E, Jeremic B. Lung Cancer in Georgia. J Thorac Oncol. 2020 Jul; 15(7): 1113-1118. doi: 10.1016/j.jtho.2020.02.030. PMID: 32593445.

15. Ahmed M. Elzawawy. Could African and Low- and Middle-Income Countries Contribute Scientifically to Global Cancer Care? JGO Journal of Global Oncology. December 2015; V1, Issue 2. DOI:10.1200/ JGO.2015.001032

16. State of Lung Cancer. American Lung Association. Key Findings. https://www.lung.org/research/state-of-lung-cancer/keyfindingslast updated: March 12, 2020.

17. GBD 2015 Risk Factors Collaborators. Global, regional, and national comparative risk assessment of 79 behavioral, environmental and occupational, and metabolic risks or clusters of risks, 1990-2015: a systematic analysis for the Global Burden of Disease Study 2015. Lancet. 2016 Oct; 388 (10053): 1659-1724

18. BREATHING IN A NEW ERA. A comparative analysis of lung cancer policies across Europe. The Economist Intelligence Unit Limited 2019 http://www.eiu.com/graphics/marketing/pdf/LungCancer\%20in-Europe-EIU-2019-9-10-final.pdf 


\section{Questionnaire}

Name/surname:

Institution:

City:

Specialty:

\section{Challenging pretreatment aspects of lung cancer in Georgia}

1. How many novel lung cancer patients do you see per month?

o $1-4$

o $\quad 5-10$

o $11-20$

o $>20$

2. Which investigation do you use for staging of lung cancer (initial staging procedures)?

(Multiple choices acceptable)

o Chest CT

o Abdominal CT

o Bronchoscopy

o Brain MRI

o Abdominal US

o Else (please specify)

3. Would you consider exploratory thoracotomy in the diagnosis and/or staging process of lung cancer?

o Always

o Never

o Occasionally (please specify)

4. In daily practice, when do you use thoracotomy for staging?

o Never

o Only when bronchoscopy or percutaneous biopsy didn't yield morphologic verification

o Pleural metastasis in suspicion

o In all lung cancer cases

5. When do you use PET-CT in NSCLC

$\mathrm{o}$ for routine staging (all patients)

o only in patients who are surgical treatment candidates

o in locally advanced, unresectable disease

o rarely

6. When do you use PET-CT in SCLC

o always for routine staging

o only in patients with LD

o in patients with ED

o rarely

7. When do you perform Brain MRI (both for NSCLC/SCLC)?

o In all patients

o In early stages (I-II)

o In locally advanced Lung cancer

o In patients with non-brain metastatic disease

o Only in symptomatic patients

o Never 
8. What is the waiting time from diagnostics to starting treatment?

$0<2$ weeks

o 2-4 weeks

o $>4$ weeks

9. Do you encourage patients to stop smoking during treatment?

o always

o sometimes

o never

10. Which molecular testing do you perform in clinical practice?

$\begin{array}{ll}\text { o } & \text { EGFR } \\ \text { o } & \text { ALK } \\ \text { o } & \text { ROS1 } \\ \text { o } & \text { PD-L1 overexpression } \\ \text { o } & \text { KRAS }\end{array}$

11. Is it important to start Lung Cancer screening in Georgia?

o Yes

o No

12. If yes, why it is important to start LC screening in Georgia? 